\title{
Identification and interaction analysis of key miRNAs in medullary thyroid carcinoma by bioinformatics analysis
}

\author{
LIJIE ZHANG $^{1}$, DONGHUI LU ${ }^{1}$, MEIQIN LIU ${ }^{1}$, MINGJIN ZHANG ${ }^{2}$ and QUAN PENG ${ }^{2,3}$ \\ ${ }^{1}$ Tumor Diagnosis and Treatment Center; ${ }^{2}$ General Surgery Department, PLA 901 Hospital, Hefei, Anhui 230031; \\ ${ }^{3}$ Pancreas Center, The Second Affiliated Hospital of Nanjing Medical University, Nangjing, Jiangsu 210003, P.R. China
}

Received December 4, 2018; Accepted June 13, 2019

DOI: $10.3892 / \mathrm{mmr} .2019 .10463$

\begin{abstract}
Medullary thyroid carcinoma (MTC) is an endocrine tumor and comprises 5-10\% of all primary thyroid malignancies. However, the biomechanical contribution to the development and progression of MTC remains unclear. In this study, To discover the key microRNAs (miRNAs or miRs) and their potential roles in the tumorigenesis of MTC, the microarray datasets GSE97070, GSE40807 and GSE27155 were analyzed. The datasets were downloaded from the Gene Expression Omnibus (GEO) database. The differentially expressed miRNAs (DEMs) and genes (DEGs) were accessed by R. Targets of DEMs and predicted using starBase, and functional and pathway enrichment analyses were performed using Metascape. A protein-protein interaction (PPI) network and an analysis of modules were constructed using NetworkAnalyst. Finally, a network was constructed to show the regulatory association between transcription factors (TFs), DEMs and downstream genes. A total of 5 DEMs were found both in GSE97070 and GSE40807, including 3 upregulated DEMs and 2 downregulated DEMs. The Gene Ontology (GO) and Kyoto Encyclopedia of Genes and Genomes (KEGG) pathway enrichment analyses from Metascape revealed that the target genes of upregulated DEMs were significantly enriched in adherens junction, kinase and protein binding, while the target genes of downregulated DEMs were mainly involved in non-canonical Wnt signaling pathway and RNA transport. From the PPI network, 13 nodes were screened as hub genes. Pathway enrichment analysis revealed that the top 5 modules were mostly enriched in the neurotrophin signaling pathway, mRNA surveillance pathway and MAPK signaling pathway. In addition, the TF-DEMs-target gene and DEGs regulatory network revealed that 17 TFs regulated 2 miRNAs,
\end{abstract}

Correspondence to: Dr Quan Peng, General Surgery Department, PLA 901 Hospital, 424 Changjiang Road, Hefei, Anhui 230031, P.R. China

E-mail: pengquan1983@163.com

Key words: medullary thyroid carcinoma, differentially expressed miRNAs, microarray analysis, bioinformatics analysis, interaction analysis including upregulated or downregulated DEMs, CREB1 regulated all upregulated DEMs, and TGFB1 was an activator of hsa-miR-199a-3p and a repressor of hsa-miR-429. Taken together, the present study identified several miRNAs and potential biological mechanisms involved in the tumorigenesis of MTC. This study identified the key DEMs and potential mechanisms underlying the development of MTC, and provided a series of biomarkers and targets for the management of MTC.

\section{Introduction}

Medullary thyroid carcinoma (MTC) arises from the calcitonin-producing parafollicular $\mathrm{C}$ cells of the thyroid and was first described by Hazard in 1959 (1). MTC comprises 5-10\% of all primary thyroid malignancies $(2,3)$. MTC is mainly sporadic (termed SMTC) and only 20-30\% of cases are hereditary MTC (HMTC) (4-6). Activating mutations in the RET proto-oncogene are responsible for medullary thyroid carcinoma, and these mutations are believed to be associated with a more persistent disease and a lower overall survival (7-9). However, to date, the molecular mechanisms of MTC carcinogenesis remain unclear.

MicroRNAs (miRNAs or miRs) are a large subgroup of non-coding RNAs, 18-25 nucleotides in length, that are evolutionary conserved. These molecules control post-transcriptional gene expression through the inhibition of mRNA translation or induction of its degradation (10) miRNAs can act as oncogenes, or tumor suppressor genes and can be used as diagnostic and predictive biomarkers, and even for the treatment of diseases, including MTC $(11,12)$. Shabani et al reported that a high expression of hsa-miR-144 and hsa-miR-183miR-34a could be considered as biomarkers of MTC (13). Furthermore, in a previous study, hsa-miR-375 was upregulated and hsa-miR-9* was downregulated in SMTC vs. HMTC, and the overexpression of hsa-miR-183 and hsa-miR-375 in MTC predicted lateral lymph node metastases (14).

Although advances have been made in understanding the mechanisms of MTC, studies on these are limited and further confirmation is required. This study aimed to identify biomarkers by analyzing differentially expressed miRNAs (DEMs) and differentially expressed genes (DEGs) between MTC and normal thyroid tissues. Subsequently, Gene Ontology (GO) and pathway enrichment analysis, protein-protein 
interaction (PPI) and transcription factor (TF)-DEMs-genes networks were conducted to identify key miRNAs and elucidate the potential molecular mechanisms of MTC.

\section{Materials and methods}

Data source. The original datasets comparing the gene expression profiles between MTC and normal thyroid tissue were downloaded from the NCBI GEO databases. The accession numbers were GSE97070 (15), GSE40807 (16) and GSE27155 (17,18). The microarray data of GSE97070 were based on GPL18402 (Agilent-046064 Unrestricted_Human_ miRNA_V19.0_Microarray), and included 8 MTC samples, 9 lymph node metastasis samples and 3 normal samples. GSE40807 was based on GPL18227 (Agilent-019118 Human miRNA Microarray 2.0 G4470B), which consisted of 40 MTC samples and 40 normal samples. The platform of GSE27155 was GPL96, [HG-U133A] Affymetrix Human Genome U133A Array, including 96 samples with 2 MTC and 4 normal samples. Platform and series matrix file(s) were downloaded as TXT files.

Data pre-processing and DEM analysis. A comparison between the 2 sample groups, MTC and lymph node metastasis vs. normal thyroid tissues, was performed in each GEO dataset to identify DEMs and DEGs. The R software package was used to process the downloaded files and to convert and reject the unqualified data, and the limma $\mathrm{R}$ package was then used to identify DEMs and DEGs. Before we decided to conduct this study, we carried out research on bioinformatics analysis, and found that the 'false discovery rate (FDR) $<0.05$, $\mid \log 2 \mathrm{FCl}>1$ ' was a common criteria for screening DEMs or DEGs (19-21). Thus, in this study, samples with the criteria mentioned above were considered DEMs or DEGs. The TXT results were preserved for subsequent analysis. Heat maps of DEMs and DEGs were generated using FunRich 3.1.3 software.

Identification of miRNA targets. starBase (http://starbase. sysu.edu.cn/index.php) provides certain miRNA-target regulatory association pairs, which are verified by experiments and predicted by 7 programs, including microT, miRanda, miRmap, PITA, RNA22, PicTar and TargetScan (22). StarBase v3.0 identifies >1.1 million miRNA-ncRNA, 2.5 million miRNA-mRNA, 2.1 million RBP-RNA and 1.5 million RNA-RNA interactions from multi-dimensional sequencing data. In this study, miRNA-target gene regulatory association pairs were verified according to the following standards: CLIP Data (low stringency), Degradome Data (low stringency), Pan-cancer (1 cancer type) and Program Number (3 programs).

GO and kyoto encyclopedia of genes and genomes (KEGG) pathway enrichment analyses of target genes. $\mathrm{GO}$ is a common method for annotating genes, gene products and sequences to underlying biological phenomena; KEGG is an integrated database resource for the biological interpretation of genome sequences and other high-throughput data (23). Metascape (http://metascape.org) is an online program that aims to develop a set of reliable, productive and intuitive tools that help biomedical research community to analysis gene/protein lists and make better data-driven decisions (24). In this study, Metascape was used to perform GO and KEGG pathway analysis on target genes of DEMs. In addition, GO terms consisted of 3 aspects: Biological process (BP), cellular component (CC) and molecular function (MF).

PPI network construction and analysis of modules. NetworkAnalyst (https://www.networkanalyst.ca/) is a series of web-based tools for statistical meta-analysis, visual data mining and data integration, through the rapid generation of biological networks. It supports the meta-analysis of gene lists, and data integration is achieved through robust statistical procedures and subsequently visually examined within PPI networks $(25,26)$. In this study, STRING was selected as a PPI database. To access more objective and reliable results, this study restricted the sources requiring experimental evidence and the cut-off score was set at a high confidence (900). Nodes with more degrees were considered as hub genes and may serve as core proteins or key candidates with important physiological regulatory functions. The pathway enrichment analysis of genes in the modules was performed, and $\mathrm{P}<0.05$ was considered to indicate a statistically significant difference.

TF-DEMs-target gene and DEGs regulatory network construction. TransmiR (http://www.cuilab.cn/transmir) is a database for TF-miR regulations, through which regulatory associations between TFs and miRNAs can be identified. To date, TransmiR v2.0 contains 3,730 literature-curated TF-miRNA regulations from 1,349 publications and 1,785,998 TF-miRNA regulations derived from ChIP-seq evidence $(27,28)$. This study inputted the overlapped DEMs into the database to examine the regulatory association pairs between TFs and DEMs. The inclusion criteria of evidence were supported by high-throughput experiments from literature.

Based on the data this study obtained, TF-DEMs-target gene and DEGs regulatory network was constructed and visualized by Cytoscape 3.6.1 software to show the overlapped TFs, DEMs, target genes and DEGs. Therefore, these TFs, DEGs, DEMs and target genes may play a potential role in the pathogenesis and treatment of MTC.

\section{Results}

Microarray data information and identification of DEMs and DEGs. The MTC expression microarray datasets GSE97070, GSE40807 and GSE27155 were standardized. The datasets were subsequently screened using the limma package $(\mathrm{FDR}<0.05, \mid \log 2 \mathrm{FCl}>1) ; 57 \mathrm{DEMs}$ were obtained in GSE97070. Among these, 29 upregulated and 28 downregulated DEMs were identified. Overall, 134 DEMs were screened from the GSE40807 dataset, including 70 upregulated genes and 64 downregulated genes. Among these, hsa-miR-375, hsa-miR-127-3p and hsa-miR-429 were significantly upregulated in both the GSE97070 and GSE40807 datasets, whereas the expression of hsa-miR-199b-5p and hsa-miR-199a-3p was downregulated. In addition, 235 DEGs were screened from the GSE27155 dataset, including 135 upregulated genes and 100 downregulated genes (Fig. 1 and Table SI). Heatmaps were generated based on the expression levels, where each column represented a biological sample 

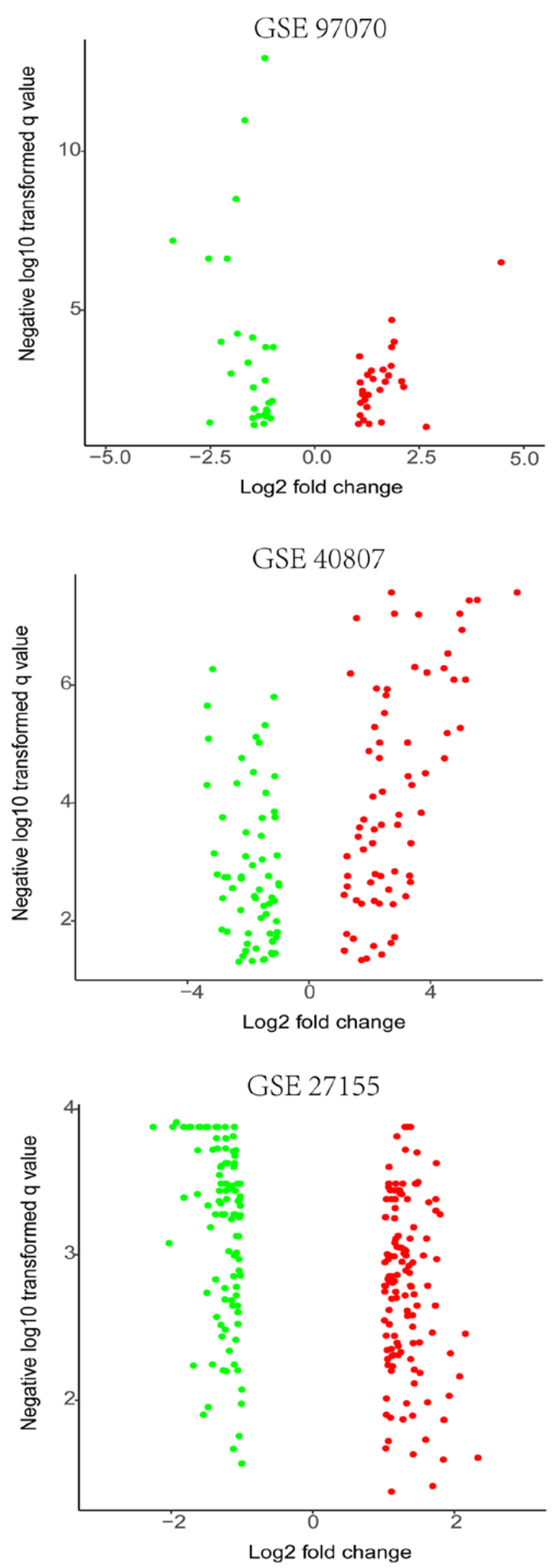

Figure 1. Differential expression of data between two sets of samples. The red points represent upregulated miRNAs or genes and green points represent the downregulation of the expression of miRNAs or genes.

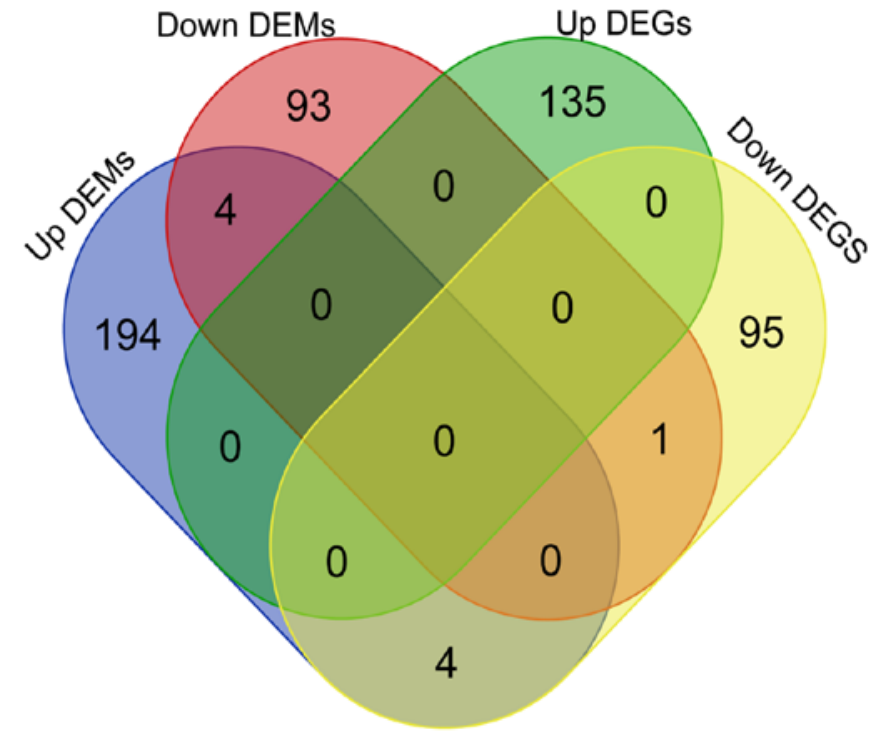

Figure 2. Venn diagram of overlapping genes between target genes of DEMs and DEGs. DEMs, differentially expressed miRNAs; DEGs, differentially expressed genes.

and each row in the heat map represented a DEM or DEG. The color indicated the relative expression levels of miRNA in tissue specimens (Figs. S1-S3).

Target genes of DEMs. The target genes of DEMs were identified according to the standards described above. For the 5 commonly altered miRNAs, a total of 300 target genes were obtained, including 202 genes of upregulated DEMs and 98 genes of downregulated DEMs (Table SII).

SNTB2 and MED13 were predicted as the potential targets of hsa-miR-375 and hsa-miR-429. A total of 4 genes were the potential targets of hsa-miR-429 and hsa-miR-199a-3p, including SCD, CFL2, IREB2 and DTNA. In addition, 5 genes, including CITED2, DUSP1, FHL1, LDHB and C1orf115, of 100 downregulated DEGs were potentially targeted by DEMs (Figs. 2 and 3).

Significant functions and pathway enrichment analysis. Metascape was used to analyze the downstream target genes of the five common DEMs. For each given gene list, a pathway and process enrichment analysis was carried out with the following ontology sources: KEGG pathway, GO $\mathrm{BP}, \mathrm{GO}$ CC and GO MF. All genes in the genome were used as the enrichment background. Terms with a P-value $<0.01$, a minimum count of 3 , and an enrichment factor $>1.5$ were collected and grouped into clusters based on their membership similarities. The enrichment factor is the ratio between the observed counts and the counts expected by chance. For GO term enrichment analysis, the target genes of upregulated DEMs were mainly enriched in adherens junction, anchoring junction and cell-substrate junction assembly, while the target genes of downregulated DEMs were mainly enriched in non-canonical Wnt signaling pathway, heat shock protein binding and regulation of small molecule metabolic process. KEGG analysis revealed that the target genes of upregulated DEMs were mostly enriched in focal adhesion, regulation of actin cytoskeleton and adherens junction. The target genes 


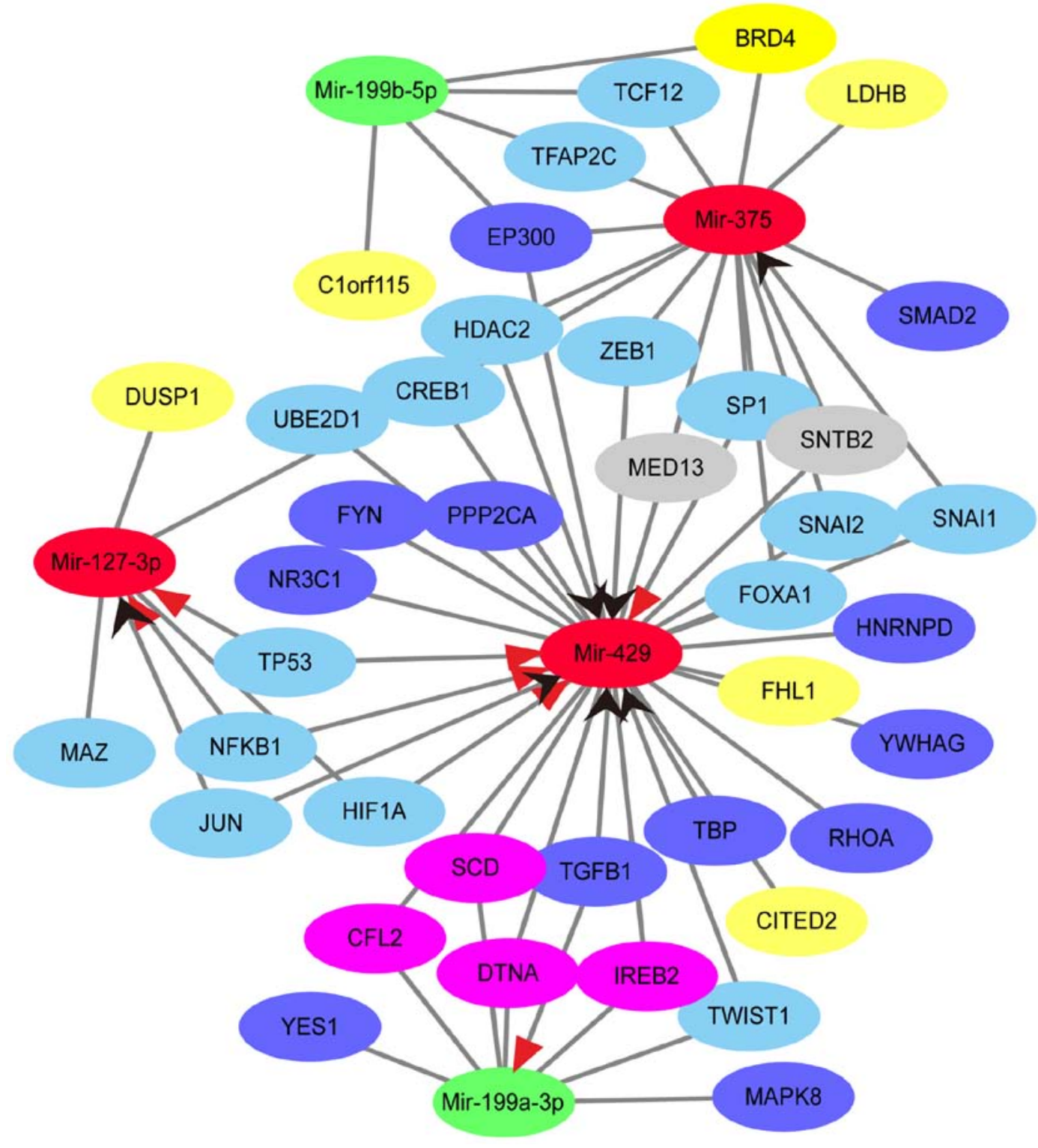

Figure 3. TF-DEMs-target genes and DEGs regulatory network. Red represents upregulated DEMs, green represents downregulated DEMs, dark blue represents hub genes, light blue represent TFs, yellow represents overlapped genes between DEGs and target genes of DEMs, pink represents overlapped genes between hsa-miR-429 and hsa-miR-199a-3p; grey represents overlapped genes between hsa-miR-429 and hsa-miR-375; red arrows indicate activation and black arrows indicate repression. TF, transcription factor; DEMs, differentially expressed miRNAs; DEGs, differentially expressed genes.

of downregulated DEMs were enriched in RNA transport, the lysosome and mRNA surveillance pathway (Table I and Fig. 4).

PPI network construction and analysis of modules. The PPI network was constructed and visualized by NetworkAnalyst. The minimum network was used to keep seed proteins, as well as essential non-seed proteins, that were suitable for simplifying a dense network to study key associations. A zero-order network was subsequently used to keep only seed proteins that directly interact with each other and to visualize modules. Degree $>20$ was set as the cut-off criterion. A total of 13 genes were chosen as hub genes, such as UBC, EP300, MAPK8, FYN, RHOA, PPP2CA, SMAD2, UBE2D1, HNRNPD, YES1, YWHAG, NR3C1 and TBP. A total of 9 genes were target genes of hsa-miR-429 (EP300, FYN, RHOA, PPP2CA, UBE2D1, HNRNPD, YWHAG, NR3C1 and TBP). In addition, the top 5 modules were selected, and the KEGG pathway enrichment analysis revealed that target genes in these modules were mostly enriched in the neurotrophin signaling pathway, the MAPK signaling pathway, the pathways in cancer, the Wnt signaling pathway and the focal adhesion (Fig. 5 and Table SIII).

Analysis of TF-DEMs-target genes and DEGs regulatory network. From the data of TransmiR, upregulated DEMs were regulated by $51 \mathrm{TFs}$, and downregulated DEMs were regulated by 24 TFs. In addition, 17 TFs regulated 2 miRNAs, including upregulated or downregulated DEMs, while CREB1 regulated all upregulated DEMs. Furthermore, EP300 was detected as a TF of hsa-miR-375 and hsa-miR-199b-5p, which was also a target gene of has-miR-429 (Table SIV). In summary, based on the aforementioned results from the bioinformatics analysis, a regulatory network was constructed to 
Table I. Results of top $10 \mathrm{GO}$ terms and top 3 KEGG pathway analysis.

\begin{tabular}{|c|c|c|c|c|}
\hline Category & Term & Description & Gene counts & $\log \mathrm{P}$ \\
\hline \multicolumn{5}{|c|}{ Target genes of upregulated DEMs } \\
\hline GO:0005912 & $\mathrm{CC}$ & Adherens junction & 20 & -7.110 \\
\hline GO:0070161 & $\mathrm{CC}$ & Anchoring junction & 20 & -6.902 \\
\hline GO:0007044 & $\mathrm{CC}$ & Cell-substrate junction assembly & 9 & -6.880 \\
\hline GO:0051347 & $\mathrm{BP}$ & Positive regulation of transferase activity & 21 & -6.251 \\
\hline GO:0005925 & $\mathrm{CC}$ & Focal adhesion & 16 & -6.123 \\
\hline GO:0005924 & $\mathrm{CC}$ & Cell-substrate adherens junction & 16 & -6.080 \\
\hline GO:0030055 & $\mathrm{CC}$ & Cell-substrate junction & 16 & -6.024 \\
\hline GO:0019900 & MF & Kinase binding & 22 & -5.909 \\
\hline GO:0034329 & BP & Cell junction assembly & 12 & -5.872 \\
\hline GO:0019904 & MF & Protein domain specific binding & 21 & -5.842 \\
\hline hsa04510 & KEGG & Focal adhesion & 12 & -6.581 \\
\hline hsa04810 & KEGG & Regulation of actin cytoskeleton & 10 & -4.637 \\
\hline hsa04520 & KEGG & Adherens junction & 6 & -4.333 \\
\hline \multicolumn{5}{|c|}{ Target genes of downregulated DEMs } \\
\hline GO:0035567 & $\mathrm{BP}$ & Non-canonical Wnt signaling pathway & 5 & -4.074 \\
\hline GO:0031072 & MF & Heat shock protein binding & 5 & -3.724 \\
\hline GO:0062012 & BP & Regulation of small Molecule metabolic process & 7 & -3.187 \\
\hline GO:0001738 & $\mathrm{BP}$ & Morphogenesis of a polarized epithelium & 4 & -3.151 \\
\hline GO:0016055 & $\mathrm{BP}$ & Wnt signaling pathway & 8 & -3.042 \\
\hline GO:0198738 & $\mathrm{BP}$ & cell-cell signaling by wnt & 8 & -3.030 \\
\hline GO:0019904 & MF & Protein domain specific binding & 10 & -3.026 \\
\hline GO:0005793 & $\mathrm{CC}$ & $\begin{array}{l}\text { Endoplasmic reticulum-Golgi intermediate } \\
\text { compartment }\end{array}$ & 4 & -2.684 \\
\hline GO:0060071 & $\mathrm{BP}$ & Wnt signaling pathway, planar cell polarity pathway & 3 & -2.599 \\
\hline GO:0090175 & $\mathrm{BP}$ & Regulation of establishment of planar polarity & 3 & -2.560 \\
\hline hsa03013 & KEGG & RNA transport & 5 & -3.037 \\
\hline hsa04142 & KEGG & Lysosome & 4 & -2.684 \\
\hline hsa03015 & KEGG & mRNA surveillance pathway & 3 & -2.132 \\
\hline
\end{tabular}

GO, gene ontology; BP, biological process, CC, cellular component; MF, molecular function; KEGG, Kyoto Encyclopedia of Genes and Genomes; DEMs, differentially expressed miRNAs.

indicate the overlapped TFs, DEMs, target genes and DEGs (Fig. 3).

\section{Discussion}

MTC is a rare malignancy with poor prognosis, as lymph node metastases are found in 55\% patients by the time of diagnosis (29). Surgical resection remains the most effective therapy of this disease, however in advanced cases and patients with distant metastases, this treatment method is not sufficient (30-33). Therefore, it is important to study the molecular mechanisms of the carcinogenesis and development of MTC.

In the present study, a bioinformatics approach was used to identify candidate biomarker and therapeutic targets of MTC. Following the analysis, 191 DEMs, including 99 upregulated DEMs and 92 downregulated DEMs were identified. Among these, hsa-miR-375, hsa-miR-127-3p and hsa-miR-429 were upregulated, and hsa-miR-199a-3p and hsa-miR-199b-5p were downregulated in 2 miRNA profiles, suggesting that they may function as carcinogens or tumor suppressors in MTC. As shown by the OncomiR database, hsa-miR-199a-3p and hsa-miR-199b-5p were upregulated in normal tissues, and hsa-miR-375 was upregulated in thyroid carcinoma, which is in accordance with the analysis of this study. Furthermore, a number of researchers have reported that the overexpression of hsa-miR-375 significantly contributes to the pathophysiology and development of MTC (34-36).

GO and KEGG analysis results showed that target genes of upregulated DEMs were significantly enriched in adherens junction and focal adhesion, which might play important roles in the tumor development and progression. Target genes of downregulated DEMs were mainly involved in Wnt signaling pathway, protein binding and RNA transport. The Wnt signaling pathway is a group of signal transduction pathways, which begins with proteins that pass signals into a cell through cell surface receptors. It is an important signaling pathway in biological development and tumorigenesis (37-39). 


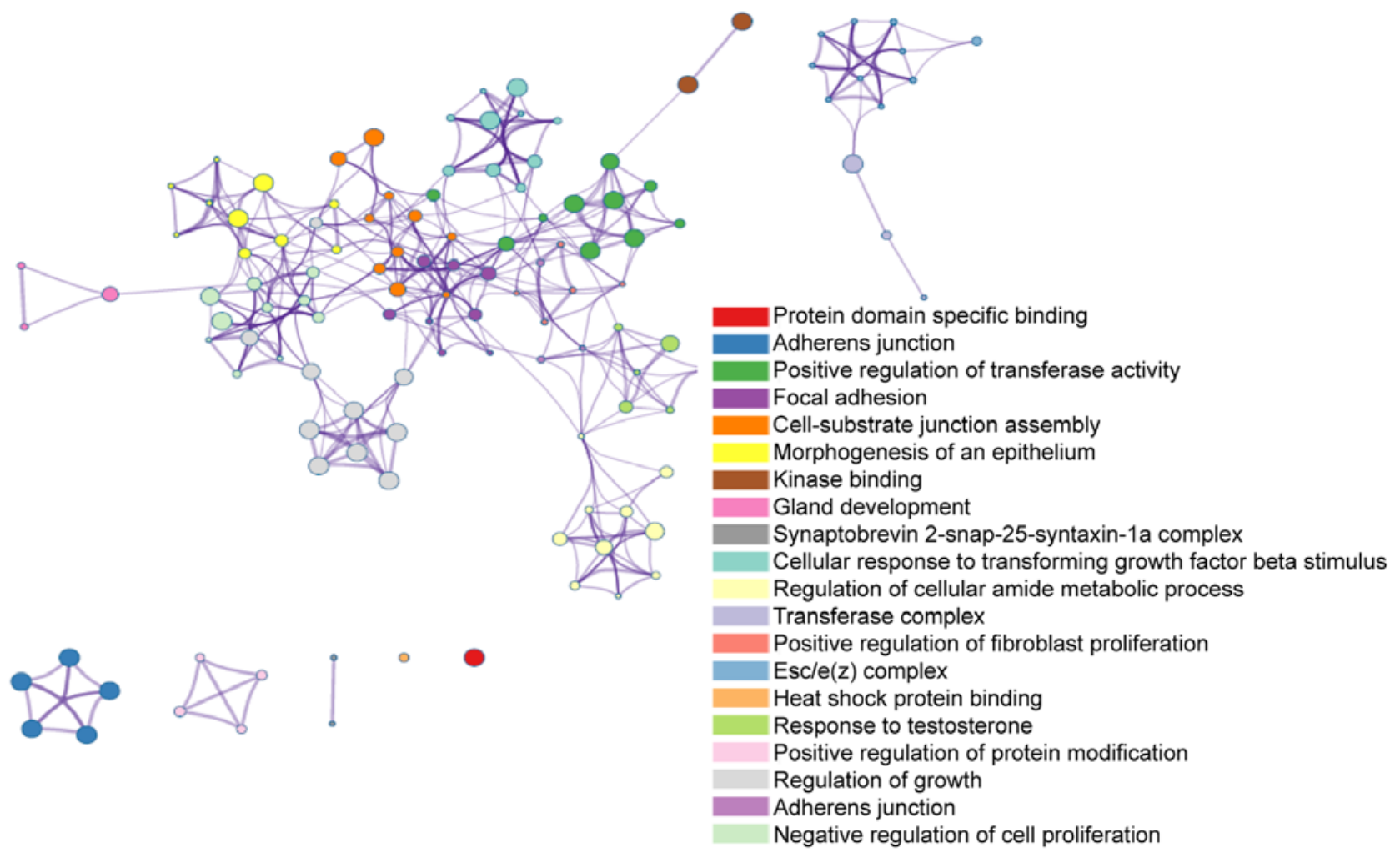

Figure 4. Top 20 clusters with their representative enriched terms (colored by cluster ID). where nodes that share the same cluster ID are typically close to each other.

In the comparison of $\mathrm{MTC}^{\mathrm{M} 918 \mathrm{~T}}$ and $\mathrm{MTC}^{634}$, in addition to MTC $^{\mathrm{M} 918 \mathrm{~T}}$ and $\mathrm{MTC}^{\mathrm{WT}}$, Maliszewska et al reported that many biochemical pathways were involved in the malignant behavior of MTC, including the Wnt pathway (40).

By constructing the PPI network, the present study identified 13 hub genes, and 10 of these, EP300, MAPK8, FYN, RHOA, PPP2CA, SMAD2, UBE2D1, HNRNPD, YES1 and NR3C1 were involved in the top 5 modules. KEGG pathway enrichment analysis of modules showed that Focal adhesion, TGF- $\beta$ signaling pathway and Wnt signaling pathway contained 4 hub genes respectively. The TGF- $\beta$ signaling pathway mediates intracellular signaling and participates in embryonic development, tumorigenesis, and physiological processes (41). Furthermore, TGF- $\beta$ can cause enhanced adhesion and motility of tumor cells (42). Santarpia et al observed the effect of miRNAs in MTC tumorigenesis, migration, proliferation and invasion. The cell lines were treated with miR-200 inhibitor and analysis was performed in accordance to the array data, showing that the members of the miR-200 family regulate the expression of E-cadherin by directly targeting ZEB1,2 and through the enhanced expression of tumor growth factor $\beta-1,2$ (43).

The hub genes MAPK8 and RHOA were involved in the 5 of the top 10 pathways, including the neurotrophin signaling pathway, MAPK signaling pathway, colorectal cancer, adherens junction, TGF-beta signaling pathway and Wnt signaling pathway. RHOA encodes a member of the Rho family of small GTPases, which cycle between inactive GDP-bound and active GTP-bound states, and function as a molecular switch in signal transduction cascades. The overexpression of this gene is associated with tumor cell proliferation and metastasis. A number of studies have suggested that RHOA can serve as a biomarker of colorectal cancer, with regards to a therapeutic target $(44,45)$. Takahashi et al conducted an experiment on Rb1(+/-)Nras(+/-) animals. The results of the aforementioned study revealed that distant MTC metastases were associated with the loss of the remaining wild-type Nras allele. In addition the loss of Nras in Rb1-deficient $\mathrm{C}$ cells results in an elevated RHOA activity, therefore leading to the malignant behavior of these cells (46). MAPK8 is a member of the MAP kinase family. The activation of this kinase by TNF- $\alpha$ is found to be required for TNF- $\alpha$ induced apoptosis. The importance of the MAPK pathway has been well established in the tumorigenesis of papillary thyroid cancer $(47,48)$. For MTC, Chang et al conducted an exome-wide analysis of the mutational spectrum and indicated that a number of pathways was involved in the variant process, including MAPK pathway (49).

In the constructed TF-DEMs-target genes and DEGs regulatory network, 5 downregulated DEGs (C1orf15, CITED2, DUSP1, FHL1 and LDHB) were also the target genes of 3 upregulated DEMs, namely hsa-miR-375, hsa-miR-127-3p and hsa-miR-429, and the downregulated DEM, hsa-miR-199b-5p. In addition, DUSP1 and FHL1 were involved in the module of PPI network, and DUSP1 participated in the MAPK pathway. The protein encoded by DUSP1 was involved in several cellular processes and resulted in chemotherapy and radiotherapy resistance, which indicated that DUSP1 can serve as a target for cancer therapy. Despite that DUSP1 has been reported as an oncogene, therapeutic target and a biomarker in many different types of tumor (50-52), the study of DUSP1 expression and function in thyroid carcinoma is limited and further investigations are required. 

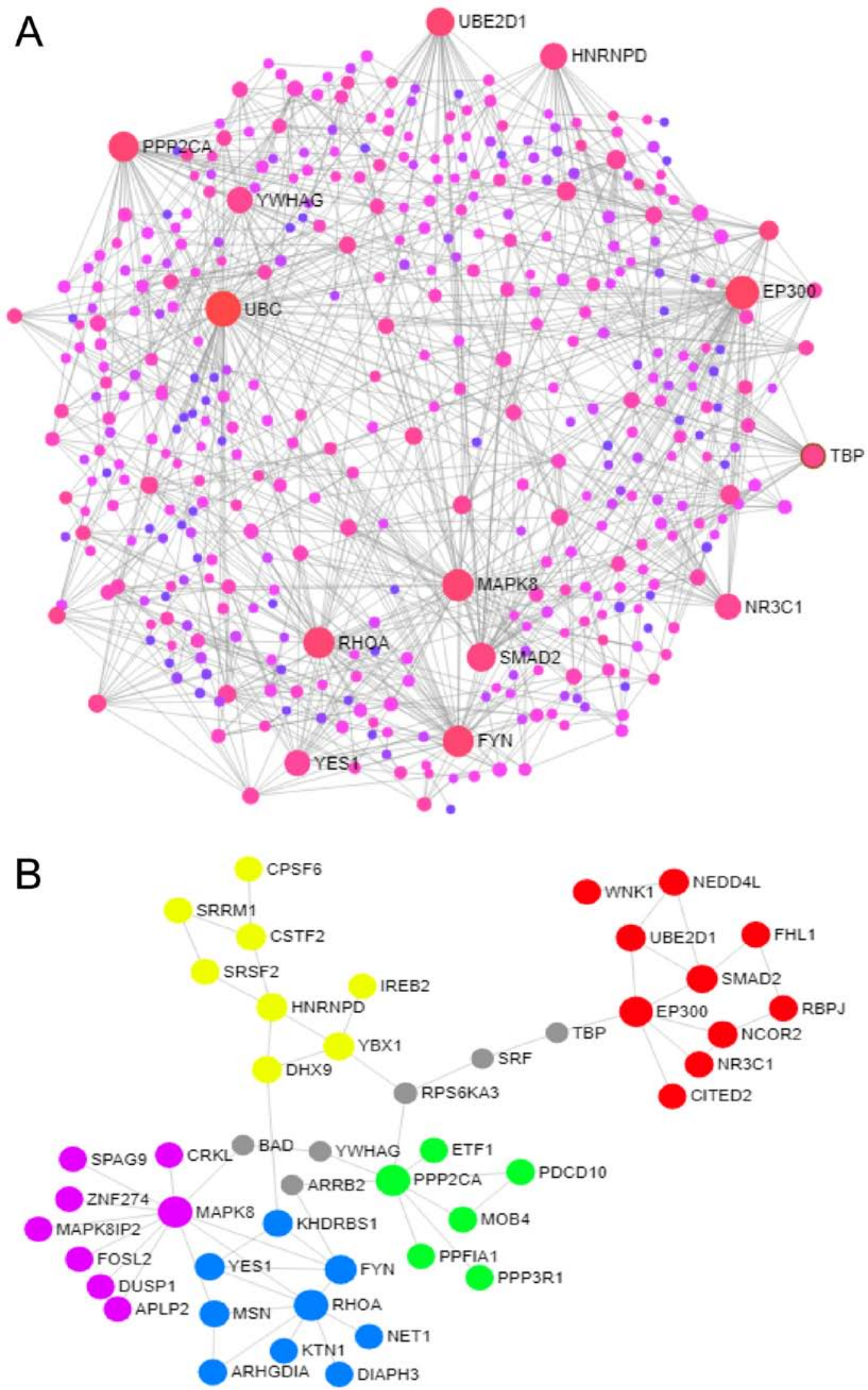

Figure 5. PPI network and the selected module. (A) PPI network from minimum network, red nodes with labels indicated hub genes; (B) top 5 significant module selected from zero-order network, each module was represented by a different color. PPI, protein-protein interaction.

As shown in the regulatory network, hsa-miR-429 and hsa-miR-199a-3p were regulated by TGFB1, a TF which encodes a secreted ligand of the TGF- $\beta$ superfamily and regulates cell proliferation, differentiation and growth. In addition, TGFB1 has been recognized as an activator of hsa-miR-199a-3p and a repressor of hsa-miR-429, based 
on the data from TransmiR by the evidence level of literature. According to the results of target prediction, 4 genes, including CFL2, DTNA, IREB2 and SCD, were overlapped between has-miR-429 and has-miR-199a-3p, whose effects have been reported in tumors for their differential expression or potential function in diagnosis and treatment (53-57). However, to the best of our knowledge, CFL2, DTNA, IREB2 and SCD has not been mentioned in MTC. In addition, 10 of the 13 hub genes were the target genes of hsa-miR-429 and hsa-miR-199a-3p, including MAPK8 and RHOA. Taken together, the regulatory network of TGFB1, hsa-miR-429/hsa-miR-199a-3p and target genes may play important roles in the development of MTC and warrant further investigation.

Cancer is a complex disease caused by multiple factors and integrated bioinformatics analysis can help in the investigation and understanding of its molecular mechanism. The aim of present study was to identify key DEMs and genes and to determine potential biomarkers to predict the progression of MTC. However, this study presents with a number of limitations. First, the dataset sample size was limited, due to the difficulty to obtain clinical samples. Second, there were only 5 DEMs that overlapped in different GSE chips, suggesting that some valuable miRNAs may be missing. Third, the incidence of MTC was low; thus, studies of how those genes affect the prognosis of MTC were seldom reported. Our team are collecting the clinical and pathological data of MTC in order to carry out further investigations.

In conclusion, this study identified numerous DEMs that may contribute to the initiation and development of MTC. Furthermore, the present study also identified a series of significant pathways and mechanisms for treatment. The regulatory association between TGFB1, hsa-miR-429 and hsa-miR-199a-3p may provide novel insight for the diagnosis and treatment of MTC. In addition, we aim to perform further experiments to examine the expression of the identified DEMS and genes in different sample types, and subsequently confirm their utility in the diagnosis and molecular therapy of MTC.

\section{Acknowledgements}

Not applicable.

\section{Funding}

No funding was received.

\section{Availability of data and materials}

The datasets used and/or analyzed during the current study are available from the corresponding author on reasonable request.

\section{Authors' contributions}

QP, DL and MZ contributed to the design of the study. LZ, ML and QP performed the bioinformatics analysis and wrote the manuscript. LZ and DL were responsible for article revision. $\mathrm{LZ}$ and ML contributed to language editing and the revision of this manuscript. All authors have read and approved the final version of the manuscript.

\section{Ethics approval and consent to participate}

Not applicable.

\section{Patient consent for publication}

Not applicable.

\section{Competing interests}

The authors declare that they have no competing interests.

\section{References}

1. Mohammadi M and Hedayati M: A brief review on the molecular basis of medullary thyroid carcinoma. Cell J 18: 485-492, 2017.

2. Figlioli G, Landi S, Romei C, Elisei R and Gemignani F: Medullary thyroid carcinoma (MTC) and RET proto-oncogene: Mutation spectrum in the familial cases and a meta-analysis of studies on the sporadic form. Mutat Res 752: 36-44, 2013.

3. Sippel RS, Kunnimalaiyaan M and Chen H: Current management of medullary thyroid cancer. Oncologist 13: 539-547, 2008.

4. Milling RV, Grimm D, Krüger M, Grosse J, Kopp S, Bauer J, Infanger M and Wehland M: Pazopanib, cabozantinib, and vandetanib in the treatment of progressive medullary thyroidcancer with a special focus on the adverse effects on hypertension. Int J Mol Sci 19 pii: E3258, 2018.

5. Fagin JA and Wells SA Jr: Biologic and Clinical perspectives on thyroid cancer. N Engl J Med 375: 1054-1067, 2016.

6. American Thyroid Association Guidelines Task Force, Kloos RT, Eng C, Evans DB, Francis GL, Gagel RF, Gharib H, Moley JF, Pacini F, Ringel MD, Schlumberger M and Wells SA Jr: Medullary thyroid cancer: Management guidelines of the American Thyroid Association. Thyroid 19: 565-612, 2009.

7. Agrawal N, Jiao Y, Sausen M, Leary R, Bettegowda C, Roberts NJ, Bhan S, Ho AS, Khan Z, Bishop J, et al: Exomic sequencing of medullary thyroid cancer reveals dominant and mutually exclusive oncogenic mutations in RET and RAS. J Clin Endocrinol Metab 98: E364-E369, 2013.

8. A T, F S, G P and M B: Genetic alterations in medullary thyroid cancer: Diagnostic and prognostic markers. Curr Genomics 12: 618-625, 2011.

9. Elisei R, Cosci B, Romei C, Bottici V, Renzini G, Molinaro E, Agate L, Vivaldi A, Faviana P, Basolo F, et al: Prognostic significance of somatic RET oncogene mutations in sporadic medullary thyroid cancer: A 10-year follow-up study. J Clin Endocrinol Metab 93: 682-687, 2008.

10. Bartel DP: MicroRNAs: Genomics, biogenesis, mechanism, and function. Cell 116: 281-297, 2004.

11. Ambros V: The functions of animal microRNAs. Nature 431: 350-355, 2004.

12. Pishkari S, Paryan M, Hashemi M, Baldini E and MohammadiYeganeh S: The role of microRNAs in different types of thyroid carcinoma: A comprehensive analysis to find new miRNA supplementary therapies. J Endocrinol Invest 41: 269-283, 2018.

13. Shabani N, Razaviyan J, Paryan M, Tavangar SM, Azizi F, Mohammadi-Yeganeh S and Hedayati M: Evaluation of miRNAs expression in medullary thyroid carcinoma tissue samples: miR-34a and miR-144 as promising overexpressed markers in MTC. Hum Pathol 79: 212-221, 2018.

14. Abraham D, Jackson N, Gundara JS, Zhao J, Gill AJ, Delbridge L, Robinson BG and Sidhu SB: MicroRNA profiling of sporadic and hereditary medullary thyroid cancer identifies predictors of nodal metastasis, prognosis, and potential therapeutic targets. Clin Cancer Res 17: 4772-4781, 2011.

15. Romeo P, Colombo C, Granata R, Calareso G, Gualeni AV, Dugo M, De Cecco L, Rizzetti MG, Zanframundo A, Aiello A, et al: Circulating miR-375 as a novel prognostic marker for metastatic medullary thyroid cancer patients. Endocr Relat Cancer 25: 217-231, 2018 .

16. Lassalle S, Zangari J, Popa A, Ilie M, Hofman V, Long E, Patey M, Tissier F, Belléannée $\mathrm{G}$, Trouette $\mathrm{H}$, et al: MicroRNA-375/SEC23A as biomarkers of the in vitro efficacy of vandetanib. Oncotarget 7: 30461-30478, 2016. 
17. Giordano TJ, Kuick R, Thomas DG, Misek DE, Vinco M, Sanders D, Zhu Z, Ciampi R, Roh M, Shedden K, et al: Molecular classification of papillary thyroid carcinoma: Distinct BRAF, RAS, and RET/PTC mutation-specific gene expression profiles discovered by DNA microarray analysis. Oncogene 24: 6646-6656, 2005.

18. Giordano TJ, Au AY, Kuick R, Thomas DG, Rhodes DR, Wilhelm KG Jr, Vinco M, Misek DE, Sanders D, Zhu Z, et al: Delineation, functional validation, and bioinformatic evaluation of gene expression in thyroid follicular carcinomas with the PAX8-PPARG translocation. Clin Cancer Res 12: 1983-1993, 2006.

19. Li D, Hao X and Song Y: Identification of the key MicroRNAs and the miRNA-mRNA regulatory pathways in prostate cancer by bioinformatics methods. Biomed Res Int 2018: 6204128, 2018.

20. Mou T, Zhu D, Wei X, Li T, Zheng D, Pu J, Guo Z and Wu Z Identification and interaction analysis of key genes and microRNAs in hepatocellular carcinoma by bioinformatics analysis. World J Surg Oncol 15: 63, 2017.

21. Li J, Qin Y and Zhang H: Identification of key miRNA-gene pairs in chronic lymphocytic leukemia through integrated analysis of mRNA and miRNA microarray. Oncol Lett 15: 361-367, 2018.

22. Li JH, Liu S, Zhou H, Qu LH and Yang JH: starBase v2.0. Decoding miRNA-ceRNA, miRNA-ncRNA and protein-RNA interaction networks from large-scale CLIP-Seq data. Nucleic Acids Res 42 (Database Issue): D92-D97, 2014.

23. Kanehisa M, Sato Y, Kawashima M, Furumichi M and Tanabe M: KEGG as a reference resource for gene and protein annotation. Nucleic Acids Res 44 (D1): D457-D462, 2016.

24. Tripathi S, Pohl MO, Zhou Y, Rodriguez-Frandsen A, Wang G Stein DA, Moulton HM, DeJesus P, Che J, Mulder LC, et al: Meta- and orthogonal integration of influenza 'OMICs' data defines a role for UBR4 in virus budding. Cell Host Microbe 18 : 723-735, 2015

25. Xia J, Benner MJ and Hancock RE: NetworkAnalyst-integrative approaches for protein-protein interaction network analysis and visual exploration. Nucleic Acids Res 42 (Web Server Issue): W167-W174, 2014.

26. Xia J, Gill E and Hancock RE: NetworkAnalyst for statistical, visual and network-based meta-analysis of gene expression data. Nat Protoc 10: 823-844, 2015.

27. Wang J, Lu M, Qiu C and Cui Q: TransmiR: A transcription factor-microRNA regulation database. Nucleic Acids Res 38 (Database Issue): D119-D122, 2010.

28. Tong Z, Cui Q, Wang J and Zhou Y: TransmiR v2.0: An updated transcription factor-microRNA regulation database. Nucleic Acids Res 47 (D1): D253-D258, 2019.

29. Scollo C, Baudin E, Travagli JP, Caillou B, Bellon N, Leboulleux S and Schlumberger M: Rationale for central and bilateral lymph node dissection in sporadic and hereditary medullary thyroid cancer. J Clin Endocrinol Metab 88: 2070-2075, 2003

30. Griebeler ML, Gharib H and Thompson GB: Medullary thyroid carcinoma. Endocr Pract 19: 703-711, 2013.

31. Schlumberger M, Carlomagno F, Baudin E, Bidart JM and Santoro M: New therapeutic approaches to treat medullary thyroid carcinoma. Nat Clin Pract Endocrinol Metab 4: 22-32, 2008.

32. Conzo G, Polistena A, Calò PG, Bononi P, Gambardella C, Mauriello C, Tartaglia E, Avenia S, Sanguinetti A, Medas F, et al: Efficacy of combined treatment for anaplastic thyroid carcinoma: Results of a multinstitutional retrospective analysis. Int J Surg 12 (Suppl 1): S178-S182, 2014.

33. Conzo G, Avenia N, Ansaldo GL, Calò P, De Palma M, Dobrinja C, Docimo G, Gambardella C, Grasso M, Lombardi CP, et al: Surgical treatment of thyroid follicular neoplasms: Results of a retrospective analysis of a large clinical series. Endocrine 55: 530-538, 2017

34. Shi L, Zhao SM, Luo Y, Zhang AW, Wei LH, Xie ZY, Li YY and Ma W: MiR-375: A prospective regulator in medullary thyroid cancer based on microarray data and bioinformatics analyses. Pathol Res Pract 213: 1344-1354, 2017.

35. Galuppini F, Bertazza L, Barollo S, Cavedon E, Rugge M, Guzzardo V, Sacchi D, Watutantrige-Fernando S, Vianello F, Mian C and Pennelli G: MiR-375 and YAP1 expression profiling in medullary thyroid carcinoma and their correlation with clinical-pathological features and outcome. Virchows Arch 47: 651-658, 2017.

36. Hudson J, Duncavage E, Tamburrino A, Salerno P, Xi L, Raffeld M, Moley J and Chernock RD: Overexpression of miR-10a and miR-375 and downregulation of YAP1 in medullary thyroid carcinoma. Exp Mol Pathol 95: 62-67, 2013.
37. Nusse $\mathrm{R}$ and Clevers $\mathrm{H}$ : Wnt/ $\beta$-catenin signaling, disease, and emerging therapeutic modalities. Cell 169: 985-999, 2017.

38. Tai D, Wells K, Arcaroli J, Vanderbilt C, Aisner DL, Messersmith WA and Lieu CH: Targeting the WNT signaling pathway in cancer therapeutics. Oncologist 20: 1189-1198, 2015.

39. Zhan T, Rindtorff $\mathrm{N}$ and Boutros $\mathrm{M}$ : Wnt signaling in cancer. Oncogene 36: 1461-1473, 2017.

40. Maliszewska A, Leandro-Garcia LJ, Castelblanco E, Macià A de Cubas A, Goméz-López G, Inglada-Pérez L, Álvarez-Escolá C, De la Vega L, Letón R, et al: Differential gene expression of medullary thyroid carcinoma reveals specific markers associated with genetic conditions. Am J Pathol 182: 350-362, 2013.

41. Colak S and Ten Dijke P: Targeting TGF- $\beta$ signaling in cancer. Trends Cancer 3: 56-71, 2017.

42. Gonzalez DM and Medici D: Signaling mechanisms of the epithelial-mesenchymal transition. Sci Signal 7: re8, 2014.

43. Santarpia L, Calin GA, Adam L, Ye L, Fusco A, Giunti S, Thaller C, Paladini L, Zhang X, Jimenez C, et al: A miRNA signature associated with human metastatic medullary thyroid carcinoma. Endocr Relat Cancer 20: 809-823, 2013.

44. Jeong D, Park S, Kim H, Kim CJ, Ahn TS, Bae SB, Kim HJ, Kim TH, Im J, Lee MS, et al: RhoA is associated with invasion and poor prognosis in colorectal cancer. Int J Oncol 48: 714-722, 2016.

45. Zhang GY, Yang WH and Chen Z: Upregulated STAT3 and RhoA signaling in colorectal cancer (CRC) regulate the invasion and migration of CRC cells. Eur Rev Med Pharmacol Sci 20: 2028-2037, 2016

46. Takahashi C, Contreras B, Iwanaga T, Takegami Y, Bakker A, Bronson RT, Noda M, Loda M, Hunt JL and Ewen ME: Nras loss induces metastatic conversion of Rb1-deficient neuroendocrine thyroid tumor. Nat Genet 38: 118-123, 2006.

47. Liu Z, Zhang J, Gao J and Li Y: MicroRNA-4728 mediated regulation of MAPK oncogenic signaling in papillary thyroid carcinoma. Saudi J Biol Sci 25: 986-990, 2018

48. Pan J, Zhang L, Xu S, Cheng X, Yu H, Bao J and Lu R: Induction of apoptosis in human papillary-thyroid-carcinoma BCPAP cells by diallyl trisulfide through activation of the MAPK signaling pathway. J Agric Food Chem 66: 5871-5878, 2018.

49. Chang YS, Chang CC, Huang HY, Lin CY, Yeh KT and Chang JG Detection of molecular alterations in taiwanese patients with medullary thyroid cancer using whole-exome sequencing. Endocr Pathol 29: 324-331, 2018.

50. Teng F, Xu Z, Chen J, Zheng G, Zheng G, Lv H, Wang Y, Wang L and Cheng X: DUSP1 induces apatinib resistance by activating the MAPK pathway in gastric cancer. Oncol Rep 40: 1203-1222, 2018.

51. Fang J, Ye Z, Gu F, Yan M, Lin Q, Lin J, Wang Z, Xu Y and Wang Y: DUSP1 enhances the chemoresistance of gallbladder cancer via the modulation of the p38 pathway and DNA damage/repair system. Oncol Lett 16: 1869-1875, 2018.

52. Zhang Y, Zhang Y, Chen M, Liu C and Xiang C: DUSP1 is involved in the progression of small cell carcinoma of the prostate. Saudi J Biol Sci 25: 858-862, 2018.

53. Wang Y, Kuramitsu Y, Ueno T, Suzuki N, Yoshino S, Iizuka N, Zhang X, Oka M and Nakamura K: Differential expression of up-regulated cofilin-1 and down-regulated cofilin-2 characteristic of pancreatic cancer tissues. Oncol Rep 26: 1595-1599, 2011.

54. Yu BB, Lin GX, Li L, Qu S, Liang ZG, Chen KH, Zhou L, Lu QT, Sun YC and Zhu XD: Cofilin-2 acts as a marker for predicting radiotherapy response and is a potential therapeutic target in nasopharyngeal carcinoma. Med Sci Monit 24: 2317-2329, 2018.

55. Liu J, Li H, Shen S, Sun L, Yuan Y and Xing C: Alternative splicing events implicated in carcinogenesis and prognosis of colorectal cancer. J Cancer 9: 1754-1764, 2018.

56. Khiroya H, Moore JS, Ahmad N, Kay J, Woolnough K Langman G, Ismail I, Naidu B, Tselepis C and Turner AM: IRP2 as a potential modulator of cell proliferation, apoptosis and prognosis in nonsmall cell lung cancer. Eur Respir J 49: pii: 1600711, 2017.

57. Siqingaowa, Sekar S, Gopalakrishnan V and Taghibiglou C: Sterol regulatory element-binding protein 1 inhibitors decrease pancreatic cancer cell viability and proliferation. Biochem Biophys Res Commun 488: 136-140, 2017.

This work is licensed under a Creative Commons Attribution-NonCommercial-NoDerivatives 4.0 International (CC BY-NC-ND 4.0) License. 